\title{
The Appropriate of Cone Depth in Loop Electrical Excision Procedure (LEEP) for Negative Pathological Margin from High Grade Precancerous Lesion of Cervix, Retrospective Study
}

\author{
Noppames Srijarusith, Nopporn Rodpenpear*
}

\begin{abstract}
Objective: To determine the appropriate cone depth for treating high grade precancerous lesions to achieve negative pathological margins of cones from LEEPs. Other factors associated with positive pathological margin were also investigated. Methods: A Retrospective study recruited 170 patients who received indications for LEEP during January 2015 to July 2020 were enrolled. The participants were operated by a single cut of LEEP and not had previously conization before. All patient data were collected into two groups, including negative and positive cone margin groups. Then, we used the cone depth by calculating from cone tissue after formalin fixation to eliminate shrinkage effect. The appropriate cut-off points for cone depth were calculated by ROC and analyzed factors that influence positive cone margin. Results: The depth of cone $(\mathrm{mm} \pm \mathrm{SD})$ of negative margin group was $8.70( \pm 3.36)$ and $6.13( \pm 2.28) \mathrm{mm}$ in positive margin group. The appropriate cut-off points for cone depth were calculated by ROC presented at resection depth of $7.21 \mathrm{~mm}$, which displayed proper cone depth with a sensitivity of $63.53 \%$ and specificity of $71.76 \%$. Elderly age (adjusted OR 1.061, 95\%CI 1.008-1.117, $\mathrm{p}=0.002$ ), number of quadrants of lesion involvement (adjusted OR 1.182, $95 \%$ CI 1.312-2.513, $\mathrm{p}=<0.001$ ) and glandular involvement (adjusted OR 3.648, 95\%CI 1.605-8.292, $\mathrm{p}=0.002$ ) were the significant risk factors for positive margin. Conclusion: The appropriate cone depth for treating high grade precancerous lesions was at least $7.21 \mathrm{~mm}$ to achieve a negative cone margin from LEEP. The significant factors associated with positive cone margin include elderly age, more quadrants of lesion involvement and glandular involvement.
\end{abstract}

Keywords: Loop electrical excision procedure- LEEP- cone depth- negative pathological margin

Asian Pac J Cancer Prev, 23 (2), 659-664

\section{Introduction}

Cervical cancer has the third highest incidence of cancer among Thai women. There are approximately 5,500 new cases per year, an estimated 11.79 cases of cervical cancer cases per 100,000 Thai population and $93.5 \%$ are found in women older than 35 to 60 years (Sangrajrang et al., 2020). However developed countries, has a much smaller incidence. The reduced incidence is due to the provision of education to the population, raised awareness by comprehensive cervical cancer screening program, effective treatment and monitoring from the onset of abnormalities with low precancerous lesions and high precancerous lesions until it progressed to cancer (Sherris, 2001). Usually, lesions preceding cervical cancer have a gradual disease progression, and cure may be possible if there are abnormalities in cytology screening (Šarenac and Mikov, 2019). Therefore, no new cervical cancer patients should occur ideally.

When the cervical cytological screening test has abnormal results, the physicians can perform additional tests to confirm diagnosis and then has perform various treatment methods. Cervical conization was the one method used to treat high grade precancerous lesion of cervix (CIN2,3) which can be performed with different technique, for example; loop electrosurgical excision procedure (LEEP), cold knife conization, and carbon dioxide laser $\left(\mathrm{CO}_{2}\right.$ laser) (Khan and Smith-McCune, 2014). In the gynecological department of HRH Princess Maha Chakri Sirindhorn Medical Center, diagnostic and therapeutic operations have performed mainly using a loop electrosurgical excision procedure (LEEP). Consequently, the excised tissue is sent to pathologist for examination of the margin and severity of lesions.

From the current study, the complication of cervical excision procedure can occur such as blood loss, infection at the excision site of cervix, cervical stenosis and long-term effects on fertility (Kietpeerakool et al., 2006). The consequent pregnancy may be increased risk for miscarriage and preterm birth (Kietpeerakool et al., 2006; Ciavattini et al., 2015; Kyrgiou et al., 2016; Liverani et al., 2016). The wide of cone did not associate 
with a bad consequent pregnancy outcome; however, this dimension depended on size and location of lesion on cervix of individual patients. Therefore, whether the lesion is large or laterally located, the wide of cone should be wider for negatively ectocervical margin. Due to the consequence pregnancy complications, many studies have been concerned to determine the cutting depth of cone excision. For example, a cervical excision greater than 15 millimeters $(\mathrm{mm})$ in depth would significantly increase preterm birth of consequence pregnancy (Castanon et al., 2014) and will affect various pregnancy complications such as spontaneous preterm birth, premature rupture of membranes, chorioamnionitis, low birth weight, admission to neonatal intensive care, and perinatal mortality (Kyrgiou et al., 2016).

Nowadays, the newest guideline to treat high grade precancerous cervical lesion had raised more awareness of the excisional procedure but still the excisional procedure has some higher benefits (Martin-Hirsch et al., 2010; Perkins et al., 2020). Therefore, the lesser cone depth for adequate treatment result should have been expected. Despite this, we still did not know the appropriate cone depth for negative endocervical and/or deep margin. According to Beyer et al., (2017) reported the cone depth by LEEP in the 10-19.9 $\mathrm{mm}$. range had a $73 \%$ chance of negative margin with $\mathrm{AUC}=0.657,79 \%$ for sensitivity and $64 \%$ for specificity. However, the previous studies did not report a clearly optimal cut-off value of the cone depth to negative cone margin. Therefore, the purpose of our study was to find the appropriate cone depth by LEEP for negative endocervical and/or deep margin and find the risk factors which might have an effect on positive endocervical and/or deep margin.

\section{Materials and Methods}

This was a retrospective study which recruited the patients who had high grade precancerous lesion of cervix (CIN 2, 3), who visited the gynecology department for diagnostic and therapeutic operations performed using a loop electrosurgical excision procedure (LEEP) at the HRH Princess Maha Chakri Sirindhorn Medical Center (MSMC) during January 2015 to July 2020. This study was approved from the institution Ethics Committee of Faculty of Medicine, Srinakharinwirot University (registry number: SWUEC-051/2563E) for research involving human subjects.

The inclusion criteria consisted of female patients who i) had abnormal cervical cytology with colposcopic directed biopsy with pathological results of CIN 2 or CIN 3 and treated by using LEEP. ii) had HSIL of cervical cytology underwent colposcopic exam and LEEP at the same time (see and treat technique). iii) who underwent LEEP for diagnosis in case of inconsistent cytological and pathological result. Exclusion criteria were defined as: i) had previously performed LEEP or other cervical conization method was done. ii) There was more than one cut of the cervix with an electric loop in the same treatment cycle iii) the pathological result showed cervical cancer and iv) the pathological results were not high grade precancerous lesions such CIN 1, cervicitis, or HPV infection. We used the maximum of cone depth by the part of gross examination in the pathological report of LEEP specimen that had a shrinkage effect from formalin fixation so we reflect the cone depth of fresh tissue by calculation. Based on Boonstra (1983) reported the shrinkage effect of fresh tissue by formalin fixation was $8 \%$. Therefore, we add $8 \%$ of depth which reported on the gross examinations after formalin fixation to reflect the true depth of fresh cone specimen. Surgical margins were considered positive when the high grade dysplasia was closer than 1 millimeter to the endocervical or deep margin, however for $>1$ millimeter distance to the lesion was considered negative (Beyer et al., 2017; Sopracordevole et al., 2019).

Sample size calculation was performed using a formula for estimating accuracy index of diagnostic studies, involving ROC analysis. The study of 109 patients published by Beyer (2017) showed the cutting depth in the $10-19.9 \mathrm{~mm}$. range had a $73 \%$ chance negative margin of cervical dysplasia with AUC $=0.657,79 \%$ of sensitivity and $64 \%$ of specificity. This study needed more preciseness. Therefore, the sample size was calculated based on an expected 0.8 of AUC. An extra $10 \%$ was added to the calculated sample size for any missing. Thus, the total sample size of at least 170 participants was needed for inclusion.

Statistical analysis was performed using STATA version 13 (StataCorp, College Station, TX, USA). The efficacy of the cone depth including sensitivity, specificity, positive predictive value, negative predictive value, and accuracy for negative cone margin at different cut-off points was examined. The receiver operating characteristic analysis (ROC) was used to find the most appropriate cutoff point of cone depth for negative cone margin. Lastly, a logistic regression model was used to analyze factors that can influence the positive cone margin. The level of significance was defined as $\alpha=0.05 \%$.

\section{Results}

The 294 patients were eligible for inclusion criteria and enrolled in this study during the period between January 2015 to July 2020 . We excluded 124 patients because they i) had previously performed LEEP or other cervical conization methods (20 patients). ii) There was more than one cut of the cervix with an electric loop in the same treatment cycle ( 24 patients) iii) the pathological result showed cervical cancer (21patients) and iv) the pathological results were not high grade precancerous lesions (59 patients). So, the total number of patients was 170 who enrolled in this study. The demographic characteristics of the both study populations are shown in table 1 . The mean $( \pm \mathrm{SD})$ age of the patients was 38.24 $( \pm 7.68)$ years in the negative cone margin group and $43.52( \pm 11.24)$ years in positive cone margin group. The majority was multiparous and had premenopausal status in both groups. The interval time (day $\pm \mathrm{SD}$ ) after diagnosing HSIL of cervical cytologic result or colposcopic exam until LEEP were $44.47( \pm 25.31)$ days in the negative cone margin group and $39.61( \pm 20.22)$ days in positive cone margin group. In the part of colposcope finding, most of the participants showed satisfactory findings and the lesion 
Table 1. Demographic Characteristics of Patients

\begin{tabular}{|c|c|c|}
\hline Characteristic & Negative cone margin & Positive cone margin \\
\hline Age (mean \pm SD) (year) & $38.24( \pm 7.68)$ & $43.52( \pm 11.24)$ \\
\hline \multicolumn{3}{|l|}{ Parity status } \\
\hline Nulliparous & 20 & 21 \\
\hline Multiparous & 65 & 64 \\
\hline \multicolumn{3}{|l|}{ Menopausal status } \\
\hline Menopause & 8 & 17 \\
\hline Pre-menopause & 77 & 68 \\
\hline Interval after diagnosis until LEEP (mean $\pm \mathrm{SD}$ ) (day) & $44.47( \pm 25.31)$ & $39.61( \pm 20.22)$ \\
\hline \multicolumn{3}{|l|}{ Satisfactory colposcopic exam } \\
\hline Satisfactory & 82 & 74 \\
\hline Unsatisfactory & 3 & 11 \\
\hline \multicolumn{3}{|l|}{ Quadrant of lesion involvement } \\
\hline 1 & 23 & 11 \\
\hline 2 & 43 & 33 \\
\hline 3 & 2 & 3 \\
\hline 4 & 17 & 38 \\
\hline Depth of cone (mean $\pm \mathrm{SD})(\mathrm{mm})$. & $8.70( \pm 3.36)$ & $6.13( \pm 2.28)$ \\
\hline \multicolumn{3}{|l|}{ Glandular involvement } \\
\hline Yes & 48 & 72 \\
\hline No & 37 & 13 \\
\hline \multicolumn{3}{|l|}{ Surgeon } \\
\hline Gynecologic oncologist & 70 & 68 \\
\hline Fellow & 10 & 11 \\
\hline Resident & 5 & 6 \\
\hline
\end{tabular}

involved at least three quadrants. For the histopathologic result, the depth of cone ( $\mathrm{mm} \pm \mathrm{SD}$ ) of the negative cone margin group was $8.70( \pm 3.36)$ and $6.13( \pm 2.28) \mathrm{mm}$ in the positive cone margin group. Most of them had no glandular involvement and the gynecologic oncologists dominated out of all the surgeons.

The level of cone depth for negative margin had presented the performance of each level as shown in table 2. From the ROC (Figure 1), a cone depth at a level of
$7.21 \mathrm{~mm}$ displayed the most appropriate for negative cone margin with a sensitivity, specificity, positive predictive value, negative predictive value and accuracy was $63.53 \%$, $71.76 \%, 69.2 \%, 66.3 \%$, and $67.65 \%$ respectively.

Concerning the secondary analysis, the association between risk factors and positive cone margin was evaluated. The result showed an age of patient, number of quadrants of lesion involvement, satisfactory colposocpic exam and glandular involvement were significant risk

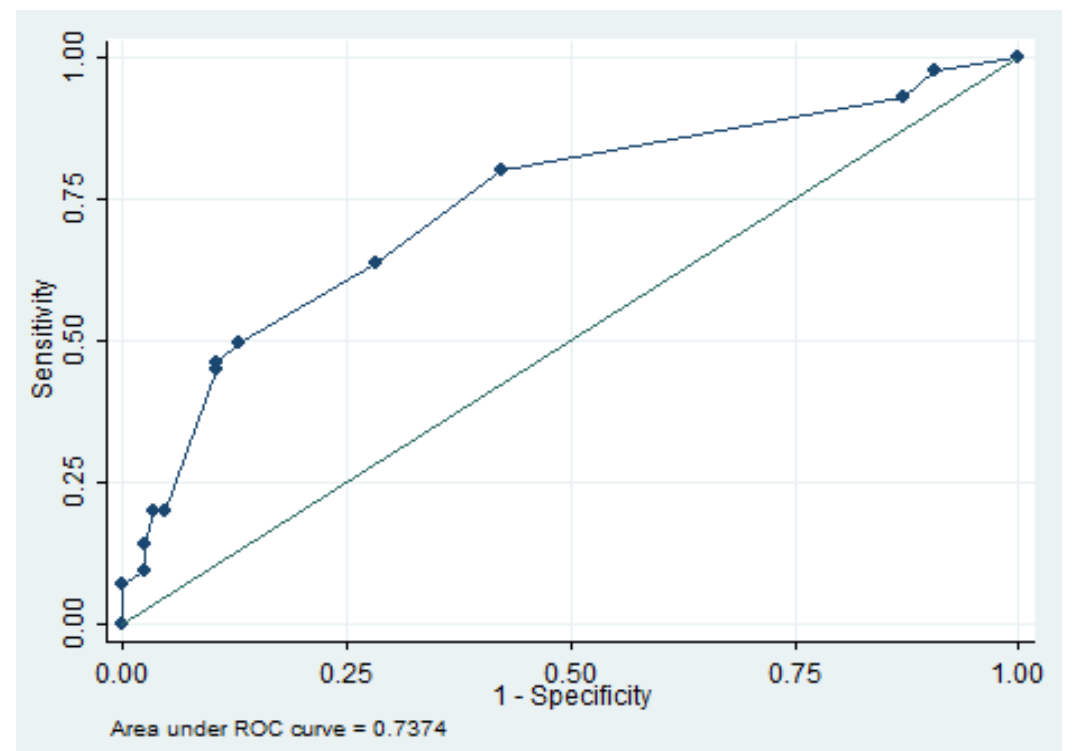

Figure 1. The Level of Cone Depth for Negative Margin 
Table 2. The Performance of Level of Cone Depth for Negative Margin

\begin{tabular}{|c|c|c|c|c|c|}
\hline Cone depth (mm.) & Sensitivity & Specificity & PPV* & $\mathrm{NPV}^{* *}$ & Accuracy \\
\hline 3.09 & $100.00 \%$ & $0.00 \%$ & - & - & $50.00 \%$ \\
\hline 4.12 & $97.65 \%$ & $9.41 \%$ & $51.90 \%$ & $80.00 \%$ & $53.53 \%$ \\
\hline 5.15 & $92.94 \%$ & $12.94 \%$ & $51.60 \%$ & $64.70 \%$ & $52.94 \%$ \\
\hline 6.18 & $80.00 \%$ & $57.65 \%$ & $65.40 \%$ & $74.20 \%$ & $68.82 \%$ \\
\hline $7.21 \dagger$ & $63.53 \%$ & $71.76 \%$ & $69.20 \%$ & $66.30 \%$ & $67.65 \%$ \\
\hline 8.24 & $49.41 \%$ & $87.06 \%$ & $79.20 \%$ & $63.20 \%$ & $68.24 \%$ \\
\hline 9.27 & $45.88 \%$ & $89.41 \%$ & $81.30 \%$ & $67.40 \%$ & $67.65 \%$ \\
\hline 10.3 & $44.71 \%$ & $89.41 \%$ & $80.90 \%$ & $61.80 \%$ & $67.06 \%$ \\
\hline 11.33 & $20.00 \%$ & $95.29 \%$ & $81.00 \%$ & $54.40 \%$ & $57.65 \%$ \\
\hline 12.36 & $20.00 \%$ & $96.47 \%$ & $85.00 \%$ & $54.70 \%$ & $58.24 \%$ \\
\hline 13.39 & $14.12 \%$ & $97.65 \%$ & $85.70 \%$ & $53.20 \%$ & $55.88 \%$ \\
\hline 14.42 & $9.41 \%$ & $97.65 \%$ & $80.00 \%$ & $51.90 \%$ & $53.53 \%$ \\
\hline 15.45 & $7.06 \%$ & $100.00 \%$ & $100.00 \%$ & $51.80 \%$ & $53.53 \%$ \\
\hline$>15.45$ & $0.00 \%$ & $100.00 \%$ & - & - & $50.00 \%$ \\
\hline
\end{tabular}

$\dagger$, the most appropriate cut-off point; *PPV, positive predictive value; * NPV, Negative predictive value

factors for positive cone margin by LEEP as shown in table 3. Finally, a multivariate logistic regression model was used. Overall, it is confirmed that elderly age of patient had $6.1 \%$ increased risk of positive cone margin (adjusted OR 1.061, 95\%CI 1.008-1.117, p=0.002), same as number of quadrants of lesion involvement had increase 18.2\% (adjusted OR 1.182, 95\%CI 1.312-2.513, $\mathrm{p}=<0.001)$ and glandular involvement had 3.648 times higher risk for having positive cone margin (adjusted OR $3.648,95 \%$ CI 1.605-8.292, $\mathrm{p}=0.002$ ) as shown in Table 4.

\section{Discussion}

High grade precancerous lesion of cervix was currently considered the precursor of invasive cervical cancer
(Sopracordevole et al, 2019). The incidence was mostly in late reproductive to premenopausal period, the disease progressed in a gradual period and easier to screening than other organs (Šarenac and Mikov, 2019). When the doctors found abnormal cytological screening results such as high grade intraepithelial neoplasia (CIN2,3) can be performed conization for treatment, especially LEEP technique (Šarenac and Mikov, 2019; Apgar et al., 2013). Several previous studies reported more deeper of cone depth than our study for negative pathological margin. Prendiville et al., (1995) found optimal LEEP cone depth of 7 to $15 \mathrm{~mm}$, Bayer et al., (2017) reported negative cone margin was achieved in $100 \%$ with a cone depth of $\geq 20 \mathrm{~mm}$ and Kyrgiou (2016) reported resection depth between $10-19.9 \mathrm{~mm}$ led to $73.0 \%$ negative cone margin.

Table 3. The Association of Risk Factors and Positive Cone Margin by Bivariate Analysis

\begin{tabular}{lccc}
\hline Factor & Crude OR & $95 \%$ CI & P value \\
\hline Age & 1.062 & $1.025-1.101$ & $0.001^{*}$ \\
Parity status & 1.291 & $0.982-1.696$ & 0.067 \\
Menopausal status & 2.406 & $0.977-5.927$ & 0.056 \\
Satisfactory colposcopic exam & 4.063 & $1.091-15.130$ & $0.037^{*}$ \\
Quadrant of lesion involvement & 1.687 & $1.271-2.239$ & $0.001^{*}$ \\
Glandular involvement & 4.269 & $2.058-8.858$ & $0.001^{*}$ \\
Surgeon & & & - \\
$\quad$ Gynecologic oncologist & 1 & - & 0.791 \\
$\quad$ Fellow & 1.132 & $0.452-2.839$ & 0.737 \\
$\quad$ Resident & 1.235 & $0.360-4.238$ & \\
\hline
\end{tabular}

* significant factor

Table 4. The Association of Risk Factors and Not Free Cone Margin by Multivariate Analysis

\begin{tabular}{lccc}
\hline Factor & Adjusted OR & $95 \%$ CI & P value \\
\hline Age & 1.061 & $1.008-1.117$ & 0.002 \\
Quadrant of lesion involvement & 1.182 & $1.312-2.513$ & $<0.001$ \\
Glandular involvement & 3.648 & $1.605-8.292$ & 0.002 \\
\hline
\end{tabular}

*, Factors that include in multivariate analysis were age, parity status, menopausal status, satisfactory colposcopic exam, quadrant of lesion involvement, glandular involvement 
In addition, Kliemann et al., (2012) reported 10 - $14 \mathrm{~mm}$ of cone depth considering the percentage chance of free margins in the order of $84-97 \%$, the likelihood of complete resection of CIN 2,3 was approximately $100 \%$ with a cone depth of $20.0 \mathrm{~mm}, 95 \%$ with a cone depth of $13.0 \mathrm{~mm}$ and $90 \%$ with a cone depth of $11.0 \mathrm{~mm}$. In the olden days, Anderson et al., (1980) reported destruction of tissue up to $3.80 \mathrm{~mm}$ in depth for eradication of $99.7 \%$ of CIN 3 lesions which had an interesting point to consider lesser cone depth. In this decade, many studies attempted to raise awareness about the depth of conization for purpose of recede complications after treatment such as bleeding, cervical stenosis, abortion and preterm birth in subsequent pregnancy (Nam, 2010; Khan and Smith-McCune, 2014; $\mathrm{Oz}$ et al, 2016). Therefore, lesser cone depth for adequate treatment should have been expected.

This study showed the performance of cone depth for negative pathological margin with the appropriate cut-off point of cone depth for negative margin was $7.21 \mathrm{~mm}$ (AUC 0.737 with $0.663-0.812$ of $95 \% \mathrm{CI}$ ) for lesser the cone depth from LEEP while the outcome of treatment for negative pathological margin was still acceptable. The reason to support our results was from Shafi et al., (2007) study, which reported CIN can involve the gland crypt of endocervix range from $1-2 \mathrm{~mm}$ to a maximum of 5.22 $\mathrm{mm}$ in depth. Therefore, the cone depth of $7.21 \mathrm{~mm}$ was adequate for negative pathological margin.

The study which reported the lesser cone depth for adequate treatment of high grade precancerous lesion as similar to our study had many studies in recently. Sopracordevole et al., (2019) reported in his study that from 1996 to 2015 showed a significant decrease in the length of cone excision over the whole study period, with a $30 \%$ reduction in the length of cone specimens. Pina et al., (2013) suggested that the shallowest LEEP that will enable complete removal of the lesion should be performed. It may be safer for women to defer pregnancy for several months after conization. Therefore, the trend of lesser cone depth was ensuring adequate treatment and should be considered.

The associated factors for positive pathological margin were elderly age (adjusted OR 1.061, 95\% CI 1.008-1.117, $\mathrm{p}=0.002$ ), more quadrants of lesion involvement (adjusted OR 1.182, 95\% CI 1.312-2.513, $\mathrm{p}<0.001)$ and the most important factor from our study was glandular involvement status (adjusted OR 3.648, 95\% CI 1.605-8.292, $\mathrm{p}=0.002$ ). This result was consistent with many previous studies (Zun et al., 2007; Kir et al., 2012; Guducu et al, 2013; Erkiling et al, 2018; Newtorn et al., 2020). Kir et al., (2021) reported in previous study, the cone depth on the patient who had a glandular involvement might deeper than without glandular involvement in purpose to negative cone margin. In the subgroup analysis of our study, the patient who had glandular involvement had the same appropriate cut-off point of cone depth for negative margin $(7.21 \mathrm{~mm})$ with sensitivity, specificity, positive predictive value and negative predictive value was $66.67 \%, 72.22 \%, 61.5 \%$ and $76.5 \%$ respectively (data shown in appendix). Therefore, even if positive glandular involvement, the cone depth might not need to be deeper than negative glandular involvement with the same level of efficacy. For the same reason, we can use the see and treat technique which omits colposcopic directed biopsy and the need to be evaluate glandular involvement on biopsy specimens before LEEP for the least of cone depth.

The strength of this study was a first study to reveal the appropriate definite cut off point of cone depth from LEEP to achieve negative pathological margins with acceptable sensitivity and specificity and appropriate sample size to a summarize the result. That supporting the treatment of patients nowadays raised awareness of the complications, especially those dealing with obstetric complications, that will occur associated with many previous studies. However, the study had limitation. Our retrospective study included patients who primarily visited gynecology department of the HRH Princess Maha Chakri Sirindhorn Medical Center (MSMC) for diagnostic and therapeutic operations performed using a loop electrosurgical excision procedure (LEEP). The majority were Thai and lived in central of Thailand e.g. Bangkok, Pathum-Thani, Nakhon -Nayok and Prachinburi province. Therefore, there was no diversity among the populations.

In conclusion, the appropriate cone depth from LEEP for treating high grade precancerous lesions was at least $7.21 \mathrm{~mm}$ to achieve negative pathological margins. In addition, the significant risk factors associated with positive pathological margin were elderly age, more quadrants of lesion involvement and glandular involvement. On the other hand, parity status, menopausal status, satisfactory colposcopic result and surgeon's experience was not a risk factor. However, the gynecologist should carefully consider the individual risk factors of each patient and concern for the minimal cone depth to optimize the treatment outcome with the least complication.

\section{Author Contribution Statement}

Conceptualization: R.N., S.N.; Data curation: R.N., S.N.; Formal analysis: R.N.; Investigation: R.N., S.N.; Methodology: R.N., S.N.; Project administration: R.N.; Resources: S.N.; Supervision: R.N.; Validation: R.N.; Visualization: R.N.; Writing - original draft: S.N.; Writing - review \& editing: R.N.

\section{Acknowledgements}

This work was supported by scholarship from Faculty of Medicine, Srinakharinwirot University, 2019-2020, Thailand. This study was approved as a part of the fellowship of gynecologic oncologist thesis (Faculty of Medicine, Srinakharinwirot University, Thailand). Furthermore, we thank Dr.Kittipong Kongsomboon, Department of Preventive and Social Medicine, Faculty of Medicine, Srinakharinwirot University, for his assistance in statistical analysis.

\section{Ethical statement}

This study was approved by the institution Ethics Committee of Faculty of Medicine, Srinakharinwirot University (Number: SWUEC-051/2563E). 


\section{Appendix}

Appendix 1: The performance of level of cone depth for negative margin in glandular involvement group.

Appendix 2: Subgroup analysis of gland involvement group (ROC).

\section{Availability of data}

The datasets that support the findings of this study are available from the corresponding author upon request.

\section{Conflict of Interest}

The authors have no conflicts of interest to declare.

\section{References}

Anderson MC, Hartley RB (1980). Cervical crypt involvement by intraepithelial neoplasia. Obstet Gynecol, 55, 546-50.

Apgar BS, Kaufman AJ, Bettcher C, Featherstone EP (2013). Gynecologic Procedures: Colposcopy, Treatment of Cervical Intraepithelial Neoplasia, and Endometrial Assessment. Am Fam Physician, 87, 836-43.

Beyer AD, Rody A, Schmidt N, Cirkel C, Neumann K (2017). Excisions of severe cervical dysplasia: Are there mandatory diameters of the cone that need to be considered?. J Turk Ger Gynecol Assoc, 18, 185-9.

Boonstra H, Oosterhuis JW, Oosterhuis AM, Fleuren GJ (1983). Cervical tissue shrinkage by formaldehyde fixation, paraffin wax embedding, section cutting and mounting. Virchows Arch A Pathol Anat Histopathol, 402, 195-201.

Castanon A, Landy R, Brocklehurst P, et al (2014). Risk of preterm delivery with increasing depth of excision for cervical intraepithelial neoplasia in England: nested casecontrol study. BMJ, 349, 6223.

Ciavattini A, Clemente N, Delli CG, et al (2015). Loop electrosurgical excision procedure and risk of miscarriage. Fertil Steril, 103, 1043-8.

Erkiling S, Taylan E, Temel O, et al (2018). Effect of endocervical glandular involvement on residual and recurrent disease in patients with high grade cervical dysplasia. Cukurova Med $J$, 43, 24-9.

Guducu N, Sidar G, Bassullu N, Turkmen I, Dunder I (2013). Endocervical glandular involvement, multicentricity, and extent of the disease are features of high-grade cervical intraepithelial neoplasia. Ann Diagn Pathol, 17, 345-6.

Khan MJ, Smith-McCune KK (2014). Treatment of cervical precancers: back to basics. Obstet Gynecol, 123, 1339-43.

Khan MJ, Smith-McCune KK (2014). Treatment of cervical precancers: back to basics. Obstet Gynecol, 123, 1339-43.

Kietpeerakool C, Srisomboon J, Khobjai A, Chandacham A, Tucksinsook U (2006). Complications of loop electrosurgical excision procedure for cervical neoplasia: a prospective study. J Med Assoc Thai, 89, 583-7.

Kir G, Karabulut MH, Topal CS, Yilmaz MS (2012). Endocervical glandular involvement, positive endocervical surgical margin and multicentricity are more often associated with high-grade than low-grade squamous intraepithelial lesion. J Obstet Gynaecol Res, 37, 1206-10.

Kir G, Karabulut MH, Yilmaz MS, Topal CS, Gocmen A (2012). Cytohistological correlation of endocervical gland involvement with high-grade squamous intraepithelial lesions. J Cytol, 29, 121-4.

Kliemann LM, Silva M, Reinheimer M, Rivoire WA, Capp E (2012). Minimal conization height for high-grade cervical squamous intraepithelial lesion treatment. Eur J Obstet Gynecol Reprod Biol, 165, 342-6.

Kyrgiou M, Athanasiou A, Paraskevaidi M, et al (2016).
Adverse obstetric outcomes after local treatment for cervical preinvasive and early invasive disease according to cone depth: systematic review and meta-analysis. $B M J, \mathbf{3 5 4}$, i3633.

Liverani CA, Giuseppe J, Clemente N, et al (2016). Length but not transverse diameter of the excision specimen for high grade cervical intraepithelial neoplasia (CIN 2-3) is a predictor of pregnancy outcome. Eur J Cancer Prev, 25, 416-22.

Martin-Hirsch PP, Paraskevaidis E, Bryant A, Dickinson HO, Keep SL (2010). Surgery for cervical intraepithelial neoplasia. Cochrane Database Syst Rev, 6, CD001318.

Nam KH, Kwon JY, Park YW (2010). Pregnancy outcome after cervical conization: risk factors for preterm delivery and the efficacy of prophylactic cerclage. J Gynecol Oncol, 21, 225-9.

Newton SDC, Andrea IF, Fernanda VF, Natasha SC, Carlos AM (2020). A review of cervical cancer precursor lesions and recurrence after treatment: What are the Real Risk Factors? J Gynecol Oncol, 3, 1029.

Oz M, Cetinkaya N, Korkmaz E, et al (2016). Optimal cone size to predict positive surgical margins after conization and the risk factors for residual disease. J Turk Ger Gynecol Assoc, 17, 159-62.

Perkins RB, Guido RS, Castle PR, et al (2020). 2019 ASCCP risk-based management consensus guidelines for abnormal cervical cancer screening tests and cancer precursors. J Low Genit Tract Dis, 24, 102-31.

Pina A, Lavallee S, Ndiaye C, Mayrand MH (2013). Reproductive impact of cervical conization. Curr Obstet Gynecol Rep, 2, 94-101.

Prendiville W (1995). Large loop excision of the transformation zone. Int J Clin Obstet Gynaecol, 38, 622-39.

Sangrajrang S, Laversanne M, Bausom R, Mery L, Bray F (2020). Cancer incidence and cancer control in Bangkok, Thailand: results from the cancer registry 2011-15 and projections to 2035. Cancer Epidemiol, 67, 1-6.

Šarenac T, Mikov M (2019). Cervical cancer, different treatments and importance of Bile Acids as therapeutic agents in this disease. Front Pharmacol, 10, 484.

Shafi MI, Welton K (2007). Colposcopy and cervical intraepithelial neoplasia. Obstet Gynaecol Reprod Med, 17, 173-80.

Sherris J, Herdman C, Elias C (2001). Cervical cancer in the developing world. West J Med, 175, 231-3.

Sopracordevole F, Carpini GD, Del Fabro A, et al (2019). Role of close endocervical margin in treatment failure after cervical excision for cervical intraepithelial neoplasia: A Retrospective Study. Arch Pathol Lab Med, 143, 1006-11.

Waxman AG, Chelmow D, Darragh TM, Moscicki A (2012). Revised terminology for cervical histopathology and its implications for management of high-grade squamous intraepithelial lesions of the cervix. Obstet Gynecol, 120, 1465-71.

Zun KH, Jun KC, Chang DY, et al (2007). LEEP conization: Risk factors of positive margin. Korean J Obstet Gynecol, 50, 1082-9.

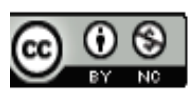

This work is licensed under a Creative Commons AttributionNon Commercial 4.0 International License. 